\title{
Rumos e Desafios: Encerrando um Processo de Avaliação da Pós-Graduação Stricto Sensu em Saúde Coletiva (1994-1997)
}

\author{
Maria Cecília de Souza Minayo ${ }^{1}$ \\ Péricles Silveira da Costa ${ }^{2}$
}

Resumo: Este artigo intenta realizar uma síntese de um processo avaliativo da Pós-Graduação (PG) em Saúde Coletiva brasileira, que vem sendo coordenado pela ABRASCO desde 1995. Particularmente, destaca questões nucleadoras de problemas identificados como prioritários para a PG da área: a estrutura curricular, analisando a possibilidade de instituição de um núcleo disciplinar ou temático para os cursos; a articulação entre os níveis formadores da PG, discutindo aspectos de ordem conceitual e organizacional; a qualidade dos cursos, buscando estabelecer parâmetros de avaliação que, ao tempo que permitam comparar os cursos, valorizem também a compreensão de suas especificidades; a produção e a divulgação científicas, numa tentativa de classificá-las e valorizá-las em suas diferentes formas. Por fim, numa perspectiva dialógica, sugere novos desafios à Pós-Graduação em Saúde Coletiva.

Palavras-chave: Avaliação; Ciência e Tecnologia; Pós-Graduação; Saúde Coletiva.

Summary: This article intends to provide a summary of an evaluation process of Post-Graduate Courses (PC) on the field of Collective Health, in Brazil, that has been carried out under ABRASCO's (Brazilian Association on Collective Health supervision, since 1995. It particularly stresses core questions of problems identified as priorities for the PC of the field: the curriculum frame, analyzing the perspective of main themes or core courses; the articulation among different PC complexity levels, reviewing conceptual and organizational issues; the courses quality, searching for evaluation parameters that whereas allows to establish comparisons among courses, also deepens the understanding of their particularities; the scientific production and publication, attempting to evaluate and qualify them in different ways. Finally, in a dialogical approach, the article suggests new challenges to the Post-graduate courses on Public Health, in Brazil.

Keywords: Evaluation Process; Science and Technology; Post-Graduation Courses; Collective Health.

\footnotetext{
1 Vice-Presidente de Ambiente, Comunicação e Informação da Fundação Oswaldo Cruz
}

2 Secretário Executivo da Abrasco 


\section{Introdução}

O processo de avaliação da Pós-Gradução stricto sensu em Saúde Coletiva desenvolveu-se durante três anos, envolvendo a auto-avaliação dos coordenadores, bem como a avaliação de todo o sistema brasileiro de Pós-Graduação stricto sensu nesta área. O processo utilizou como fonte os dados fornecidos pela tradicional avaliação promovida bianualmente pela CAPES, informações adicionais oferecidas pelos programas e incluiu a comparação do desempenho nacional com o internacional, por meio de consultorias de professores/pesquisadores nacionais e estrangeiros. A investigação, que teve o apoio da CAPES e do CNPq, foi coordenada pelos autores do presente artigo e contou com a participação permanente de coordenadores de Pós-Graduação, pesquisadores e professores da área. As atividades se encontram descritas em artigos publicados na revista Ciência E Saúde Coletiva, da ABRASCO (II[1/2], 1997), onde são descritos os pressupostos e os objetivos do processo até seu ponto culminante, em setembro de 1996, quando se organizou um Seminário para apresentação dos resultados, com a presença de todos os coordenadores da Pós-Graduação, consultores nacionais e internacionais.

$O$ presente artigo tenta sintetizar um momento posterior, decorrente do citado Seminário, quando foram detectados pontos merecedores de uma reflexão mais aprofundada e específical focalizando assuntos considerados nucleadores de problemas, levantados pelo processo avaliativo. No relatório final do evento (1997), resultado das plenárias e dos trabalhos de grupo empreendidos para discutir e propor encaminhamentos, conseguiu-se resumir os temas em quatro eixos nucleadores.

O primeiro tema diz respeito a uma tensão existente na organização dos currículos dos cursos de Pós-Graduação, provocada pelas dificuldades em delimitar o conceito de saúde. Por abranger processos biológicos, sociais, políticos e ambientais, o conceito enseja uma ampliação dos objetos com os quais se pode trabalhar cientificamente, com conseqüências sobre a organização das estruturas curriculares, tornando-as muito diversificadas e diferenciadas. Portanto, o problema inicial pode ser enunciado da seguinte forma: ter ou não ter um núcleo comum de disciplinas ou temas que possam ser apresentados e exigidos para a criaçâo de currsos novos e naqueles já existentes.

O segundo também concentra questões de ordem conceitual e organizacional. Diz respeito às formas tradicionais com que a área organiza os seus processos de formação de recursos humanos que, por terem objetivos diferenciados, propiciarem bastante liberdade acadêmica na formulação de conteúdos e serem muito diversificadas e não articuladas, têm apresentado certas irracionalidades, tais como superposição de programas, inadequação nos possíveis encadeamentos e repetição de informações, nem sempre tratadas de forma mais aprofundada que em níveis anteriores. Isso redunda, entre outros problemas, em um tempo demasiadamente longo dedicado à formação acadêmica. Os termos nucleadores poderiam, portanto, ser assim resumidos: como distinguir e articular as modalidades de formação pós-graduada.

O terceiro se refere à avaliação da qualidade dos cursos, o que implica tanto uma visão complexa dos mesmos - entendê-los como detentores de historicidade, tempos, condições e circunstâncias diferenciadas -, como a consideração de que tais cursos englobam temas teórico-conceituais e também práticos. Trata-se, em síntese, de estabelecer parâmetros que, ao mesmo tempo, preservem a comparabilidade e sejam capazes de dimensionar, de forma abrangente, os processos específicos, valorizando as diferenças e os esforços de cada um.

Por fim, o último nucleador estaria resumindo e problematizando as dificuldades para valorar os processos de produção e de divulgação científica, levando em conta a necessidade de estimular e mesmo induzir produtos 
científicos e tecnológicos que atendam as necessidades do setor, sem, de outro lado, ficar à margem das formas tradicionais de avaliação de desempenho. A questão poderia ser assim enunciada: como classificar a produção acadêmica, valorizando não apenas os produtos finais já consagrados pelos veículos tradicionais, mas também tomando uma posição ativa para estabelecer critérios, estimular e valorizar aqueles que se voltam para a prestação de serviços, para a elaboração de normas técnicas e outras tecnologias essenciais ao setor saúde.

Para analisar os pontos mencionados, foram realizadas quatro oficinas de trabalho, com a participação de profissionais interessados em discutir os temas. A primeira teve a coordenação de Everardo Duarte Nunes e Péricles Silveira da Costa; a segunda, de Paulo Elias; a terceira, de Maurício Lima Barreto e a quarta, de Moisés Goldbaum. Por fim, no V Congresso Brasileiro de Saúde Coletiva, realizado em Águas de Lindóia, em agosto de 1997, organizou-se uma oficina-síntese, visando a finalizar o processo de avaliação iniciado no IV Congresso ocorrido em Recife, em junho de 1994.

As conclusões dessa segunda etapa da avaliação estão descritas a seguir. É evidente que todo o desenvolvimento de trabalhos como o que ora se encerra levanta novos problemas e leva a adotar uma postura dinâmica de provisoriedade e vigilância sobre as ocorrências que possam aportar novidades ou riscos. Porém, é também fundamental que se tenha a justa dimensão de um momento que se esgota, para que passos à frente possam ser dados já a partir de novo patamar. É nesse sentido que aqui se coloca um ponto final que, ao mesmo tempo, enseja novo recomeço.

\section{Ter ou não ter um núcleo comum}

Observa-se na área de Saúde Coletiva um movimento, a um só tempo, de expansão e de especialização. A expansão se dá por meio de disciplinas de caráter filosófico e metodológico e de uma imensa variedade de objetos e temáticas. Esse fenômeno evidencia pujança da área, mas também revela problemas de delimitação no âmbito dos saberes e das práticas, bem como dispersão significativa nos modelos organizativos. Ou seja, se, por um lado, a excessiva liberdade na elaboração dos conteúdos e das ementas disciplinares favorece a expressão criativa dos professores/pesquisadores, por outro, provoca dificuldades de demarcar tecnicamente tais conteúdos.

Essa questão ensejou, reiteradamente, discussões epistemológicas e teóricas fundamentais sobre o conceito de saúde, carregado no aspecto das suas representações de problemas de ordem médica e de saúde pública (em sentido estrito), que se assentam nos processos de adoecimento. $\mathrm{O}$ mencionado conceito inclui, também, as condições, as concepções, as políticas e as práticas que possibilitam à sociedade ser saudável. Portanto, o objeto da Saúde Coletiva move-se entre o pensar e o fazer setorial e, como práxis da própria sociedade, pode ser, de forma aproximada, apreendido teoricamente, por meio da articulação entre a Epidemiologia, as Ciências Sociais e Humanas e Ambientais, as políticas sociais em geral e as setoriais em particular, e as práticas de promoção, prevenção e recuperação da saúde. Desta forma, o conceito de saúde é compreendido, para o desenho das estruturas curriculares, como um objeto interdisciplinar e estratégico cuja meta é gerar conhecimentos e tecnologias que possam impactar positivamente a realidade sanitária do País.

Reafirmando postura já assumida por representantes da área em outros momentos históricos, optou-se, aqui também, por não delimitar um núcleo comum de disciplinas consideradas obrigatórias para todos. Isso se deve, principalmente, ao reconhecimento de que os diversos recortes e formatos são, no momento atual, saudáveis para o avanço 
acadêmico dos programas. Por exemplo, faz parte hoje da história e da riqueza da área, e de seu compromisso com a realidade, que os cursos da Escola Nacional de Saúde Pública (ENSP) se organizem em oito subáreas de concentração, a saber: Ciências Sociais e Saúde; Epidemiologia; Grandes Endemias; Engenharia Sanitária; Políticas públicas; Planejamento e Administração; Saúde do Trabalhador; Toxicologia ambiental e ocupacional. O mesmo pode ser dito das áreas da Faculdade de Saúde Pública: Epidemiologia; Nutrição; Saúde ambiental; Serviços de saúde pública; Administração hospitalar; Saúde Materno-infantil.

Por outro lado, é fundamental, para o atendimento da demanda hoje existente, que os cursos do Instituto de Saúde Coletiva da Bahia, de Medicina Preventiva da USP/SP e da USP/RP e o do Instituto de Medicina Social da UERJ continuem com as áreas de concentração tradicionais, ou seja, as de Epidemiologia, Ciências Sociais e Planejamento. Já os cursos novos, talvez por dificuldades em compor um quadro mais amplo de docentes e pesquisadores, preferem preservar sua qualidade seguindo a tendência de serem temáticos, ora concentrando-se na Epidemiologia, ora em Políticas de saúde, ora em Saúde e Ambiente, ora em grupos populacionais específicos. Cada um desses formatos traz uma história, um movimento interno de expressão de necessidades e de adequação institucional para dar respostas e, portanto, uma contribuição peculiar, desaconselhando qualquer tentativa de enquadramento e homogeneização. Os componentes das oficinas preferiram enfrentar essa questão e, à guisa de sugestão, elaboraram algumas diretrizes a serem levadas em conta por todos os cursos, nos seus formatos organizacionais de conteúdos curriculares. São elas:

(1) um recorte conceitual e histórico que inclua:

- história e cultura da Saúde Pública/ Coletiva;
- saúde enquanto tema relevante para a sociedade e objeto das Ciências Sociais e Humanas;

- saúde enquanto objeto de políticas públicas;

- processos e perfis de adoecimento enquanto objeto da Epidemiologia;

- saúde enquanto pratica de promoção, prevenção, recuperação e organização de serviços assistenciais.

(2) um recorte técnico-instrumental que enfoque:

- metodologias quantitativas e qualitativas e conhecimentos básicos para aplicá-las.

(3) um recorte de formação geral que contemple:

- dimensões filosófica e humanística;

- liderança em C\&T e do setor saúde;

- informação e informática;

- proficiência em alguma língua estrangeira;

- atualização permanente.

Mais do que um "pacote", os itens citados constituem uma tentativa de orientação, de tal forma que, de um lado, não se deixe a área absolutamente sem limites; de outro, permitindo que se busquem formas adequadas ao perfil e à ênfase temática de cada curso. É preciso que as lideranças da área tenham em mente também que, nos tempos atuais, quando as informações são muito rapidamente superadas pelas novas tecnologias e pelas transições políticas que afetam profundamente a área da Saúde Coletiva, as ênfases devem ser dadas a processos formativos, culturais (da área) e técnicos que, simultaneamente, preservem as tradições, desafiem o que necessita ser superado e ousem inovar.

Ainda como recomendação da Oficina, foram apresentadas sete temáticas discutidas em reunião conjunta $\mathrm{CNPq} / \mathrm{ABRASCO}$, realizada em dezembro de 1996, em São Paulo, dentro da mesma ótica de avaliação e estabelecimento de prioridades. Foram elas ainda 
incluídas como termos de referência para os núcleos e centros de pesquisa em Saúde Coletiva que, conforme conhecimento público e notório, estão geralmente abrigados nos programas de Pós-Graduação. Essas indicações poderiam servir, ao mesmo tempo, como orientação para as ementas das disciplinas e para seminários nos centros de estudos dos cursos. São elas:

- Impacto da transição epidemiológica sobre a sociedade

Trata dos determinantes e dos processos das mudanças dos perfis de morbi-mortalidade, tais como queda da mortalidade infantil, comportamento das doenças infecciosas, aumento das enfermidades neuropsíquicas e crônico-degenerativas decorrentes do envelhecimento da população; bem como o reflexo dessas mudanças na utilização e adequação dos serviços e no consumo de medicamentos.

- Estratégias de redução das desigualdades em saúde

Diz respeito à relação entre classes sociais, condições de vida e trabalho e perfil de morbi-mortalidade; assim como ao acesso aos serviços dos diferentes grupos sociais, visando à superação das situações adversas.

- Modos de vida, estilos de vida e vulnerabilidade

Refere-se à descrição, compreensão e análise das questões de caráter sociocultural que expressem modos de viver a saúde e a doença, individual e coletivamente, buscando entender comportamentos saudáveis e de risco, para articular estratégias de promoção, prevenção e recuperação da saúde.

- Efeitos da globalização da economia sobre a saúde

Aborda os processos socioculturais que afetam a saúde, concomitantes à globalização econômica (urbanização acelerada, terceirização, desemprego estrutural, subemprego, autonomização da força de trabalho, migração, exclusão e marginalização, violência social). Inclui também os efeitos sobre a saúde como as doenças cardiovasculares, as ocupacionais, mentais, ambientais e as epidemias emergentes e reemergentes.

- Qualidade da gestão e aplicação dos recursos em saúde

Trata do impacto - sobre a saúde e sobre os serviços - da incorporação de tecnologias, dos recursos terapêuticos e novas metodologias, visando a conter os desperdícios e o mau aproveitamento dos recursos financeiros e a contribuir para uma melhor apropriação e adequação tecnológicas. Igualmente trata de estudos sobre financiamento do setor, produtividade, satisfação de usuários, assim como da formação de recursos humanos para o Sistema Único de Saúde (SUS).

- Comunicação e informação para a promoção da saúde

Refere-se aos estudos e programas voltados para novas tecnologias em educação e saúde, bem como para a difusão de informações técnico-científicas entre os diferentes atores e grupos sociais, pelos vários veículos de comunicação.

- Modelos organizacionais e sistemas de saúde

Abrange questões trazidas pelos processos de descentralização e outras formas organizacionais do sistema, relacionando o público e o privado e as diferentes modalidades de oferta de serviços. Estudos sobre as cooperativas, consórcios, seguro-saúde, aprimoramento dos sistemas para monitoramento de doenças e agravos e qualidade de vida.

Como se pode depreender dessas propostas, o que se pede aos cursos é que fundamentem sua organização acadêmica em sintonia com as necessidades de saúde da população brasileira; que tomem como base de seus projetos a dinâmica do seu perfil epidemiológico. Ademais, que debatam problemas estruturais e conjunturais que configuram as condições para uma sociedade saudável, buscando construir propostas que permitam enfrentar os problemas contemporâneos do País. 
Articulação entre os niveis da formação pós-graduada

O processo de avaliação constatou vários problemas conceituais e organizacionais também na oferta de cursos da área. O mais importante deles foi a evidência de um certo paralelismo entre os vários níveis, tornandoos por vezes repetitivos e antieconômicos, tanto para os sujeitos como para as instituições.

Há várias questões diagnosticadas. Uma delas foi evidenciada pelo estudo da demanda e dos egressos, mostrando uma tendência cada vez mais acentuada de diferenciação de perfil entre os estudantes de mestrado e doutorado: $63 \%$ dos primeiros são provenientes dos serviços de atenção e $26,5 \%$ são docentes e pesquisadores buscando aprimoramento. No doutorado, 68\% vêm das universidades e centros de pesquisa, em oposição aos $26 \%$ de profissionais de práticas assistenciais e de promoção. No entanto, muitos cursos continuam a tratar de forma semelhante essas clientelas distintas, sobretudo, privilegiando conteúdos acadêmicos em detrimento dos componentes tecnológicos.

Outro ponto foi a constatação da crise da "residência médica em medicina preventiva" e a ausência de diretrizes da área sobre esse ponto. Essa crise, que já levou ao fechamento de algumas delas e à baixíssima demanda de outras, está sendo enfrentada pela abertura de residências multiprofissionais em vários centros de formação, essas sim, em expansão. Na dinâmica dessa problemática, surge também a proposta da CAPES de mestrado profissional, como alternativa para áreas que tenham forte componente tecnológico; e ainda, dentro do próprio setor saúde, a demanda de treinamento para o programa "médico de família". Essa última proposta não é menos complicada, pois interage igualmente com áreas clínicas.

Na verdade, a discussão da modalidade de formação "residência em medicina preventiva ou saúde pública" ainda não está suficientemente aprofundada pela ABRASCO, e vem se prolongando há alguns anos. Certamente a crise anuncia várias questões, como a desvalorização econômica e de status do médico sanitarista; a superação do paradigma preventivista; assim como a dificuldade das instituições formadoras de responderem, no ritmo necessário, às necessidades do momento presente. Mas anuncia, também, um certo dinamismo do sistema na incorporação do conceito ampliado de saúde, quando se abre, com sucesso, para a multiprofissionalidade.

Outra face da crise se apresenta nos cursos de especialização, quase todos pensados a partir de um sistema de saúde pública centralizado e vertical. Muitos cursos tradicionais perderam clientela, demandando uma política mais clara de adequação dos centros formadores às mudanças estruturais que se processam no SUS e às demandas sociais. As novas exigências postas pelo SUS, corroboradas pela revolução dos meios de comunicação, informação e informática, pedem novos formatos, mais ágeis e mais técnicos, sob risco dos existentes se tornarem, primordialmente, apenas degraus para a Pós-Graduação stricto sensu.

Acentuaram também a crise das "residências" e das "especializações" as adaptações dos mestrados a formatos mais rápidos, mais curtos e mais técnicos, buscando atender tanto a necessidades do mercado como a précondições das instituições de fomento para outorga de bolsas e financiamentos. Por causa disso e pela oportunidade de sistematização de experiências e obtenção de bolsas, muitos candidatos, que antes buscavam cursos lato sensu, se dirigem hoje diretamente para os cursos stricto sensu.

Diante desses problemas, pode-se perceber que urge a necessidade de discussão não só dos objetivos e da articulação entre todos os níveis de formação, como da pertinência das modalidades hoje existentes e das alternativas de organização. No momento presente, o doutorado tem sua organização e seu escopo menos problemáticos, porque melhor defini- 
dos. No entanto, mesmo nesse patamar, reconhecida sua função primordialmente acadêmica - de geração de ciência e tecnologia -, é importante recomendar fortemente o acesso direto de candidatos com clara demonstração de vocação científica, sem necessidade de percorrer a longa carreira de residência, especialização e mestrado. A adoção dessa possibilidade ainda constitui exceção nos vários programas.

Levando-se em conta a relevante presença de profissionais dos serviços nos mestrados, entende-se que esse nível tenha que se programar para atender a um duplo direcionamento. De um lado, que seja tratado como um curso terminal, atendendo às necessidades desse tipo de clientela, contemplando uma formação humanística, histórico-conceitual e instrumental, ao mesmo tempo em que seu produto final seja voltado para analisar problemas do mundo real da Saúde Coletiva, enfrentados pelos sanitaristas e gestores. De outro, que seja tratado como um patamar para a carreira acadêmica, com um programa de formação que contemple os elementos já citados e, ainda, exigindo-se para titulação uma dissertação científica ou a publicação de artigos em periódicos reconhecidos na área. Sendo assim, grosso modo, o primeiro estaria mais voltado para o desenvolvimento tecnológico; e o segundo, mais dedicado ao campo científico. No momento presente, as exigências da Lei de Diretrizes e Bases (LDB) tornam urgente a necessidade de titulação de um grande número de professores universitários, o que deverá provocar, nos próximos anos, um aumento substancial da demanda aos mestrados, por parte desse grupo específico, que configura hoje um perfil intermediário entre os dois anteriores: não serão necessariamente pesquisadores em sentido pleno e nem "pensadores" dos serviços. Levantamos a hipótese de que tal situação tenderá a se acomodar na medida em que as propostas da LDB forem se concretizando na prática.
Em resumo, a relação das especializações (incluindo-se aí a residência) com a Pós-Graduação stricto sensu deve ser problematizada, questionando-se sempre as finalidades de um e de outro estágio. A formação lato sensu, para ser eficiente e eficaz, necessitaria: (a) ter uma orientação claramente voltada para a clientela; (b) estar tecnicamente capacitada para responder aos problemas concretos do campo da Saúde Coletiva; (c) deixar de ser pré-requisito para o mestrado e para o doutorado. Isso não impede, no entanto, que sejam aproveitados créditos de cursos de especialização para os níveis stricto sensu, sob a condição de serem obedecidas exigências acadêmicas.

\section{Qualidade dos cursos}

Este tema mereceu especial atenção em todos os debates sobre a Pós-Graduação, por vários motivos. Primeiro, porque a avaliação da CAPES, que pontua e classifica os cursos, determina seus níveis de qualidade acadêmica a partir da quantificação de alguns indicadores, realizando assim uma saudável competição entre os programas. Segundo, porque o processo avaliativo liderado pela ABRASCO pretendeu apontar critérios mais qualitativos, levando a área a pensar na sua especificidade, na sua diferenciação interna, no impacto de sua produção sobre os serviços e sobre os indicadores de saúde regionais e nacionais.

O crescente número de cursos novos, em condições consideradas precárias pelos avaliadores, foi também outro tópico de atenção. Existe uma certa pressão externa, sobretudo do MEC, para que as universidades criem programas de Pós-Graduação, implantando núcleos e linhas de pesquisa, a fim de que sejam reconhecidas por mérito e recebam financiamento público. Essa pressão tem grande impacto sobretudo nas instituições fora do eixo das capitais das regiões Sudeste e 
Sul e em centros universitários privados que, por várias razões, acumulam sérios problemas de adequaçào e qualidade dos cursos. A abertura de um programa de Pós-Graduação em Saúde Coletiva, em tais circunstâncias, começa a ser uma tentação fácil para as universidades, porque sua implantação não exige grandes investimentos materiais. Por outro lado, essa aparente facilidade encobre muitas dificuldades, já hoje enfrentadas por vários cursos novos. Assim, tais cursos ressentem-se de: maior número de professores fixos no Programa; falta de infra-estrutura para pesquisas; inexistência de massa crítica para compor equipes; sobrecarga sobre os titulados dessas universidades quanto ao número de horas-aula e outras atividades administrativas na graduação; e falta de cultura acadêmica, impedindo ou dificultando a dedicação às atividades científicas imprescindíveis à qualidade dos cursos.

Entretanto, o problema maior, que deve ser colocado como sinal de alerta para a área, é a tentativa de reunir docentes de várias formaçôes e especialidades - que tocam marginalmente o objeto Saúde Coletiva, pelo fato de serem titulados - para comporem o quadro docente da Pós-Graduação. Esta questão é tão séria que algumas propostas não apresentam sequer um docente de tradição na área. No caso, faltam à equipe criadora do programa conceitos teóricos básicos, visão histórica e política e "cultura" em Saúde Coletiva, comprometendo, assim, a formação específica. Talvez seja este o ponto que requeira, hoje, maior vigilância, esperando-se, por parte das lideranças desse campo do conhecimento, uma atitude que, sem ser discriminadora, forneça parâmetros para seu crescimento. Nesse sentido, a oficina-síntese faz algumas recomendações que, apesar de afetarem a todos os membros da comunidade científica da área, dirigem-se especificamente à ABRASCO que tem, por ofício, protagonizar os rumos de desenvolvimento do campo. Recomenda-se à sua diretoria que invista na institucionalização de uma avaliação permanente, tendo em conta que a qualidade está ancorada em três parâmetros: (a) na estruturação intrínseca dos cursos, cujo quadro de referência é dado pelas exigências básicas para funcionamento que se encontram nos requisitos obrigatórios para reconhecimento pela CAPES; (b) na qualidade institucional da universidade ou centro de pesquisa onde se realizam os cursos, incluindo-se aí o reconhecimento acadêmico do corpo docente; (c) no critério de relevância social no âmbito nacional ou regional para a existência dos mesmos. Tais parâmetros podem ser desdobrados em itens mais concretos, assinalados a seguir.

- Proporcionar infra-estrutura para funcionamento dos cursos, como indicador do compromisso institucional das universidades e institutos que abrigam ou pretendem abrigar programas de Pós-Graduação;

- Organizar grupos competentes de docentes/pesquisadores, exigindo sempre que neles se incluam profissionais titulados na área, considerando como imprescindiveis profissionais formados em Saúde Pública e especialistas nas disciplinas consideradas essenciais: Epidemiologia, Ciências Sociais e Saúde, Políticas públicas e de saúde, Planejamento e Administração dos serviços;

- Os cursos existentes ou a serem criados devem se assentar sobre grupos de pesquisa em Saúde Coletiva, pelo menos em alguma área de concentração específica do campo;

- Os cursos devem ser conduzidos buscando associar, na formação, ciência, tecnologia e política, tratando essas inter-relações como intrínsecas ao campo. Ou seja, a formação em Saúde Coletiva não pode ser apresentada como um processo meramente técni$\mathrm{co}$, nem como um mero repasse de conhecimentos;

- Sinalizar afirmativamente, para ser valorizado, o componente tecnológico do campo, pouco realçado, até então, quando 
se comparam as ênfases sobre os produtos tradicionais no campo científico, tais como artigos, livros e outros. Na Saúde Coletiva é preciso sublinhar a importância de estimular a dedicação na construção de normas técnicas (o que exige domínio científico), metodologias, modelos, patentes, softwares, e técnicas e produtos pedagógicos.

- Valorizar também os investimentos e avanços dos programas sobre si mesmos, tomando em conta sua história e suas condições objetivas;

- Incentivar - e se possível promover parcerias, estágios inter-cursos (nas temáticas em que cada um apresente excelência acadêmica) para estudantes e docentes, e quaisquer outras iniciativas coletivas, criando-se assim uma rede de economia de esforços, mútua valorização e reconhecimento.

\section{Produção e divulgação científicas}

Existe um consenso que, como qualquer outra área científica, a de Saúde Coletiva se referencia aos indicadores universais de reconhecimento acadêmico, por meio das publicações em periódicos indexados nacionais e internacionais e em livros com corpo editorial. É também opinião da maioria que não se deva, em área como a nossa, tão estratégica para a situação sanitária do País, enfatizar apenas publicações no exterior para se medir o impacto da produtividade dos docentes e pesquisadores. Entende-se, sim, que se deva trabalhar em uma perspectiva também qualitativa, capaz de criar parâmetros para se distinguir o mérito acadêmico e a relevância social, valorizando ambos, ainda que os tratando como diferentes.

No caso dos periódicos nacionais é importante levar-se em conta, para divulgação dos artigos, a recente categorização realizada pelo CNPq/Finep/Fapesp, na qual a Revista de Saúde Pública e os Cadernos de Saúde Pública foram pontuados em primeiro lugar em nossa área, seguindo-se História, Ciência e Saúde e Physis, classificadas como em processo de amadurecimento. O grupo de avaliação recomenda que Saúde em Debate e Saúde e Sociedade esforcem-se por manter a periodicidade e corpo editorial, bem como por ampliar as bases de indexação, de forma a constituirem espaços de divulgação com reconhecimento científico. Ultimamente, a ABRASCO lançou Ciência E Saúde Coletiva, e está no prelo a Revista Brasileira de Epidemiologia, possibilitando assim que a comunidade científica tenha várias alternativas para divulgar sua produção. As duas últimas, ainda não indexadas, encontram-se em fase de discussão e construção de identidade próprias, demandando esforço coletivo para que se aprimorem e se tornem veículos de referência.

Em relação à divulgação em livros, os avaliadores recomendam que se dê relevância aos que são frutos de investigações e que tenham corpo editorial acadêmico, concedendo-se prioridade aos publicados por editoras universitárias. Essa categoria de produto é particularmente importante para a área de Ciências Sociais, na qual o livro representa, via de regra, o cume de uma produção acadêmica.

A valorização da divulgação científica em congressos mereceu um cuidado especial na reflexão dos componentes da oficina, tornando-se consensual que a apresentação de trabalhos nos Congressos de Saúde Coletiva, Epidemiologia e Ciências Sociais, entre outros, deveriam receber alguma pontuação. A ênfase maior mereceria ser dada aos congressos e seminários temáticos organizados mais especificamente para o avanço do conhecimento. Neste sentido, sugere-se à ABRASCO que exija trabalhos completos como requisito para valorar a participação no processo avaliativo. O grupo não conseguiu avançar muito em indicações precisas sobre este tema, que constitui, portanto, um ponto de pauta para a continuidade da reflexão. 
Não menos importante foi a reiterada ênfase à necessidade de se sugerir - aos órgãos de fomento e às comissões da área a criação de indicadores para novas formas de produção existentes hoje na comunidade científica nacional e internacional, propiciadas pelos avanços dos meios comunicacionais e informacionais. Esses produtos emergentes se multiplicam e na verdade questionam os formatos tradicionais que remontam ao século XIX, quando se processou uma divisão rígida entre ciência, técnica e arte. Hoje, freqüentemente, há produtos tecnológicos (inclusive na área de Saúde Coletiva) que fazem a síntese entre os três componentes de expressão simbólica da humanidade. Como classificá-los e valorizá-los? Embora objeto de reflexão, tal ponto também não ficou suficientemente aprofundado.

Em síntese, em relação à produção científica, reafirmou-se o que já é tradicional e universalmente reconhecido, ou seja, como tratar a produção já concluída e divulgada. Sublinhou-se, ainda, a necessidade de se investir na avaliação dos docentes como orientadores: tempo de titulação dos orientandos, qualidade das teses, ritmo de atividades e abandono dos cursos pelos pós-graduandos. Para estímulo da comunidade, lembrou-se que os estudos cientométricos revelam forte tendência de crescimento da produção da área, cujo aumento, de 1990 a 1995, foi de 155\%. Observou-se uma certa endogenia nas iniciativas de divulgação, privilegiando-se veículos nacionais e os mais próximos aos docentes e pesquisadores. Da mesma forma, assinalou-se muita dispersão na escolha dos periódicos $\mathrm{e}$ recomendou-se maior esforço para intercâmbio e parceria internacionais, na tentativa de ampliar a disseminação da produção brasileira.

\section{Conclusões}

Este artigo buscou traduzir a finalização de um processo de inflexão da área de Saúde
Coletiva sobre seu desempenho na dinâmica da Pós-Graduação brasileira. Seu objetivo, muito pontual, de constituir-se ao mesmo tempo em registro e em instrumento de trabalho, pode tê-lo tornado por demais fenomênico, até mesmo descontextualizado dos graves problemas político-institucionais que a área vivencia hoje no cenário da ciência e da tecnologia nacional. Foi uma escolha escrevê-lo assim, julgando prestar uma colaboração aos que virão e darão continuidade à dinâmica de construção dos programas considerados mais avançados de formação de recursos humanos que a Saúde Coletiva vem construindo: sua Pós-Graduação. É preciso repetir que se trata de um processo exitoso, vivo, dinâmico, com tendências de aprofundamento e expansão, bem como portador de profunda relevância para a população brasileira e para o campo científico. Porém, como toda criação viva, apresenta problemas $e$ merece cuidados. Por isso, no Seminário-síntese foi elaborada uma sugestão à diretoria da ABRASCO para que institua nas rotinas da Associação um seminário anual dos coordenadores da Pós-Graduação. A idéia é que uma pequena equipe seja a condutora desse processo, diferenciado dos formatos das comissões, a ser organizado, a cada ano, por um dos programas locais.

Em toda a trajetória do trabalho os professores, pesquisadores e consultores envolvidos consideraram de fundamental importância o método de avaliação, que combinou o olhar interno e o olhar externo, ambos necessários para preservar a especificidade de cada processo local e a crítica de especialistas, no sentido de proceder às mudanças necessárias. Enalteceram o ganho em qualidade e nas relações de intercâmbio, parcerias e solidariedade entre os cursos, pela construção de um espaço que foi ao mesmo tempo democrático, orientador, solidário e exigente.

Pensando que todo fim é também recomeço, pode-se dizer que a comunidade científica da área de Saúde Coletiva, liderada pela 
ABRASCO, continua hoje, na Pós-Graduação, com pelo menos três desafios mapeados:

- Intensificar a criação de critérios de avaliação de sua produção que valorizem tanto a relevância acadêmica quanto a social. Ambas são fundamentais à área, mas devem ser diferenciadas e tratadas diferentemente;

- Definir de forma clara o que é produção tecnológica em Saúde Coletiva. Em um campo tão crucial para a transformação so-

\section{Documentos que serviram de base à presente síntese}

BARreto, M. L. (1997) - Qualidade da Pós-Graduação em Saúde Coletiva: Avaliação da Área versus Articulação dos Programas. In: Minayo, M.C.S. \& Costa, P.S. (orgs.) - Avaliação da PósGraduação Stricto Sensu em Saúde Coletiva no Brasil. Relatório complementar. Rio de Janeiro: ABRASCO, mimeo.

ELIAS, P.E.M. (1997) - Diferenciação e Articulação de Níveis da Pós-Graduação em Saúde Coletiva. In: Minayo, M.C.S. \& Costa, P.S. (orgs.) Avaliação da Pós-Graduação Stricto Sensu em Saúde Coletiva no Brasil. Relatório complementar. Rio de Janeiro: ABRASCO, mimeo.

GOLDBAUM, M. (1997) - Produção e Divulgação cial, é preciso aprofundar esse ponto para que possa ser suficientemente valorizado $\mathrm{e}$ incentivado;

- Definir e questionar sempre as prioridades para investigação, mantendo a necessária tensão entre estudos básicos, estratégicos e operacionais; entre o desenvolvimento disciplinar e a construção interdisciplinar voltada para temáticas e problemas considerados relevantes;

Científicas. In: Minayo, M.C.S. \& Costa, P.S. (orgs.) - Avaliação da Pós-Graduação Stricto Sensu em Saúde Coletiva no Brasil. Relatório complementar. Rio de Janeiro: ABRASCO, mimeo.

MINAYO, M.C.S. \& COSTA, P.S. (orgs.) (1997) Avaliação da Pós-Graduação Stricto Sensu em Saúde Coletiva no Brasil. Relatório complementar. Rio de Janeiro: ABRASCO, mimeo.

NUNES, E.D. \& COSTA, P.S. (1997) - Estrutura Curricular/Núcleo Básico Comum. In: Minayo, M.C.S. \& Costa, P.S. (orgs.) - Avaliação da PósGraduação Stricto Sensu em Saúde Coletiva no Brasil. Relatório complementar. Rio de Janeiro: ABRASCO, mimeo. 